\section{Forschung und Bildung}

gültigen EU-Wasserrahmenrichtlinie einen grundlegenden Richtungswechsel in der europäischen Wasserpolitik zu vollziehen. Insbesondere das angestrebte integrierte Flusseinzugsgebietsmanagement wirft aus sozioökonomischer Sicht viele offene Forschungsfragen auf.

Auf einem Workshop des Umweltforschungszentrums Leipzig-Halle (UFZ) Ende letzten Jahres wurde zu den Forschungsfragen der Wasserrahmenrichtlinie, den Möglichkeiten ihrer Umsetzung, sowie zu den bestehenden Mängeln der neuen EURichtlinie ein sozioökonomisches Forschungsnetzwerk Flusseinzugsgebietsmanagement und Sozioökonomie ins Leben gerufen. Dieses Netzwerk hat sich zum Ziel gesetzt, als Diskussionsforum für disziplinübergreifende Forschungsfragen zum Flusseinzugsgebietsmanagement zu fungieren, Informationen über Forschungsergebnis- se und -vorhaben auszutauschen sowie Verbundprojekte zu initiieren, um die Umsetzung der Richtlinie wissenschaftlich zu begleiten.

Kontakt: Dr. Frank Messner, UFZ Leipzig-Halle, Abteilung ÖKUS, Postfach 2, 04301 Leipzig, Tel. 0341/ 235-2204, Fax -2511, E-mail: messner@alok.ufz.de. Die Ergebnisse des Workshops sind im UFZ-Bericht 30/1999 publiziert worden und werden auf Anfrage kostenlos versandt.

\section{- Neve Praktikumsplattform Umweltpsychologie}

Seit Anfang dieses Jahres gibt es im Bereich Umweltpsychologie eine Praktikumsplattform. Diese Plattform richtet sich an Studierende der Psychologie sowie an Unternehmen, Behörden und Verbände, bei denen die Rolle des Menschen bei der Planung und Umsetzung von Umweltschutzmaßnahmen von besonderer Bedeutung ist. Psychologische Erkenntnisse finden beispielsweise im Rahmen der Umweltbildung, des Marketings ökologischer Produkte und
Dienstleistungen oder bei der wissenschaftlichen Evaluation von Umweltschutzmaßnahmen Anwendung. Die Vermittlung der Praktikumsstellen übernimmt die Initiative Psychologie und Umweltschutz (IPU). Für Praktikumsanbieter, die selbst keine Diplom-PsychologInnen beschäftigen, organisiert die IPU auch die fachliche Begleitung des/der PraktikantIn. Es stehen externe Diplom-PsychologInnen zur Verfügung, die den PraktikantInnen zur Seite stehen und so eine qualitativ hochwertige Arbeit garantieren.

Kontakt: IPU e.V., Andy Schulz, c/o Humboldt Universität zu Berlin, Institut für Psychologie, Oranienburger Str. 18, 10178 Berlin, Tel. 030/51065346 (Do 10-12 Uhr): E-mail: info.ipu@psychologie.hu-berlin.de, Internet: http://ipu.umweltpsychologie.de

\title{
Macht EMAS einen Unterschied?
}

\section{Der Treibhauseffekt ist seit Jahren eines der am meisten diskutierten globalen Umweltprobleme. Inwieweit spiegelt sich dies in der Umweltberichterstattung von Unternehmen wider? Und spielt es eine Rolle, ob sich ein Unternehmen am EG-Öko-Audit-System (EMAS) beteiligt oder nicht? Aufschluss gibt eine Analy- se deutscher Umwelterklärungen und -berichte anhand von Indikatoren zu Kohlendioxidemmissionen und zum Energieverbrauch.}

$\mathrm{D}$ Von Nicole Hroch und Stefan Schaltegger ie Umweltberichterstattung deutscher Unternehmen hat in den letzten Jahren deutlich zugenommen. Für Unternehmen, die sich an EMAS beteiligen, ist die Veröffentlichung einer Umwelterklärung Pflicht. Aber auch Unternehmen ohne EMAS-Zertifikat erstellen inzwischen häufig Umweltberichte. Eine vor kurzem abgeschlossene empirische Untersuchung analysierte über 1550 deutsche Umwelterklärungen und -berichte bezïglich zweier zentraler Fragestellungen (1):

1. Inwieweit nehmen Unternehmen auf aktuelle umweltpolitische Diskussionen Bezug? Als Beispiel wurde der Treibhauseffekt gewählt.

2. Inwiefern unterscheiden sich die Resultate zwischen Unternehmen, die sich an EMAS betei- ligen, und solchen, die zwar einen Umweltbericht, jedoch kein EMAS-Zertifikat haben?

Die untersuchten Kriterien für Kohlendioxidemmissionen und Energieverbrauch und die Ergebnisse der Untersuchung sind in der Tabelle auf S. 6 dargestellt.

\section{Wenig Informationen zu CO2}

Wie die Ergebnisse der Untersuchung zeigen, nahmen die deutschen Umwelterklärungen und -berichte wenig Bezug auf die aktuelle umweltpolitische Diskussion. Besonders hinsichtlich des Kriteriums Kohlendioxidemissionen wurde deutlich, dass nur relativ wenige Unternehmen diese Problematik im Rahmen ihrer Umweltpolitik behandeln und Maßnahmen zur Reduktion von Kohlendioxidemissionen festlegen. Dies ist gerade im Hinblick auf die 1996 von den deutschen Wirtschaftsverbänden formulierte Absichtserklärung zur Reduzierung der spezifischen Kohlendioxidemissionen um 20 Prozent von 1990 bis zum Jahr 2005 ein überraschendes Ergebnis. Verhältnismäßig am häufigsten wurden Angaben zur absoluten Höhe der Emissionen gemacht sowie Zeitreihen für die (absoluten) Emissionen dargestellt.

Die Ergebnisse zeigen weiterhin, dass die Firmen zum Energieverbrauch, insbesondere zu konkreten Energiezielen, deutlich mehr Angaben machen. Allerdings wurde der absolute Energieverbrauch nur von rund 20 Prozent der Unternehmen angegeben. In den meisten Fällen wurden statt dessen die einzelnen Energieträger aufgelistet, ohne den Gesamtverbrauch als einheitliche Größe anzugeben.

\section{Unternehmen mit EMAS-Zertifikat hinken nach}

Die Untersuchung kommt bei der zweiten Fragestellung zum Ergebnis, dass Unternehmen mit EMAS-Zertifikat bezüglich der Publikation fast aller Kohlendioxid-Indikatoren schlechter abschneiden als Unternehmen mit Umweltbericht aber ohne EMAS-Zertifikat. Dies zeigt der Vergleich der Spalten zwei und drei in Tabelle 1. 


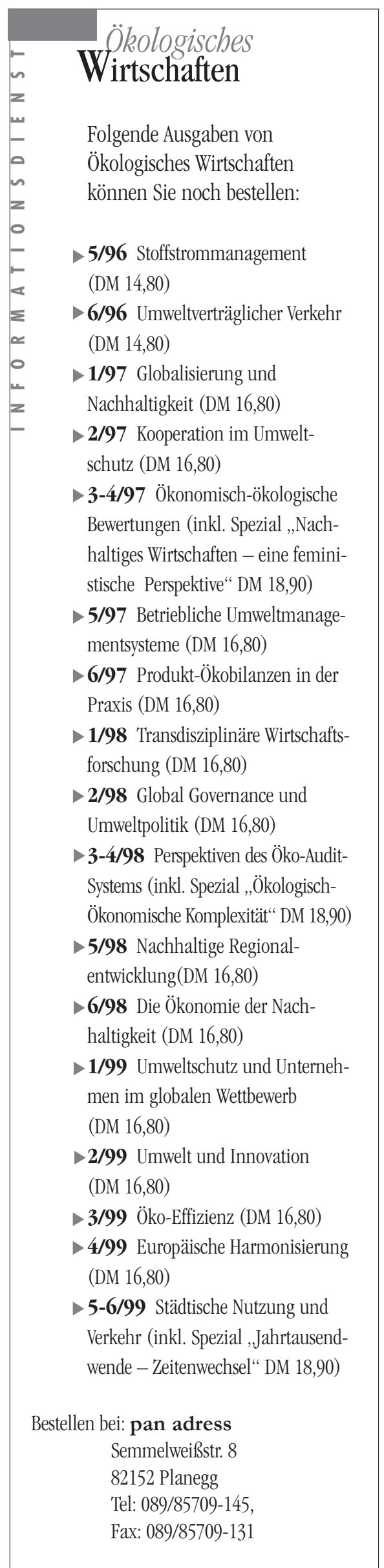

\begin{tabular}{|c|c|c|c|c|c|}
\hline Veröffentlichung & $\begin{array}{l}\text { Umwelte } \\
\text { (EMAS-Ze }\end{array}$ & $\begin{array}{l}\text { erklärung } \\
\text { rtifikat) }\end{array}$ & $\begin{array}{l}\text { Umwe } \\
\text { lohne U }\end{array}$ & $\begin{array}{l}\text { bericht } \\
\text { US-Zert.) }\end{array}$ & $\begin{array}{l}\text { Gesamt } \\
(553)\end{array}$ \\
\hline Kriterium & total 498 & in $\%$ & total 55 & in $\%$ & in $\%$ \\
\hline CO2-Ziele in der Umweltpolitik & 2 & $0,4 \%$ & 0 & $0 \%$ & $0,36 \%$ \\
\hline Konkrete CO2-Ziele & 9 & $1,81 \%$ & 6 & $10,91 \%$ & $2,71 \%$ \\
\hline Meilensteine C02-Ziele & 0 & $0 \%$ & 1 & $1,82 \%$ & $0,18 \%$ \\
\hline Absolute CO2-Emissionen & 185 & $37,15 \%$ & 29 & $52,73 \%$ & $38,70 \%$ \\
\hline CO2-Kennziffern & 17 & $3,41 \%$ & 19 & $34,55 \%$ & $6,51 \%$ \\
\hline Zeitreihen CO2-Emissionen & 77 & $15,46 \%$ & 25 & $45,45 \%$ & $18,44 \%$ \\
\hline Totale Anzahl von Angaben zu CO2 & 290 & $9,71 \%$ & 80 & $21,22 \%$ & $11,15 \%(2)$ \\
\hline Konkrete Energieziele & 176 & $35,34 \%$ & 13 & $23,64 \%$ & $34,18 \%$ \\
\hline Meilensteine Energieziele & 5 & $1,00 \%$ & 1 & $1,82 \%$ & $1,08 \%$ \\
\hline Absoluter Energieverbrauch & 86 & $17,27 \%$ & 17 & $30,91 \%$ & $18,63 \%$ \\
\hline Energie-Kennziffern & 118 & $23,70 \%$ & 23 & $41,82 \%$ & $25,50 \%$ \\
\hline Zeitreihen Energieverbrauch & 102 & $20,48 \%$ & 29 & $52,73 \%$ & $23,69 \%$ \\
\hline Totale Anzahl von Angaben zu Energie & 487 & $19,56 \%$ & 83 & $30,18 \%$ & $20,61 \%$ \\
\hline
\end{tabular}

Bei den Energieinformationen ist der Unterschied etwas weniger stark ausgeprägt, wobei Unternehmen mit EMAS-Zertifikat nur hinsichtlich konkreter Energieziele besser dastehen.

Besonders in den Umwelterklärungen werden diejenigen Indikatoren am häufigsten erfïllt, die entweder aus den Vorgaben der ,guten Managementpraktiken" (Anhang I D der EG-Öko-AuditVerordnung) oder den Responsible-Care-Grundsätzen übernommen wurden. Auch die in der EG-Öko-Audit-Verordnung genannten Indikatoren finden sich häufig. Beispielsweise fordert EMAS eine zusammenfassende Schadstoffbilanz, zu der natürlich die absoluten Kohlendioxidemissionen gehören, sowie explizit Angaben zum Energieverbrauch (3). Informationen, die über diese Anforderungen hinausgehen, drohen ungeachtet ihrer gesellschaftlichen und politischen Aktualität kaum beachtet zu werden.

Mögliche Gründe für die Unterschiede mögen in der unterschiedlichen Firmengröße oder Branchenzugehörigkeit oder einer unterschiedlich großen Erfahrung der betrachteten Unternehmen mit der Umweltberichterstattung liegen.

Das Ergebnis deutet vorderhand jedoch auch darauf hin, dass die Standardisierung des Umweltmanagements und der Umweltberichterstattung gemäß EMAS nicht (oder noch nicht) maßgeblich zu einer an der aktuellen gesellschaftlichen Diskussion orientierten proaktiven Umweltkommunikation beiträgt. Für eine abschließende Bewertung zur Aktualität der deutschen Umwelterklärungen und -berichte und der Wirkungen von EMAS sind allerdings Untersuchungen anhand weiterer Indikatoren und über einen längeren Zeitraum notwendig (4).

\section{Anmerkungen}

Quelle: eigene Darstellung

(1) Aus der Grundgesamtheit der zum Untersuchungszeitpunkt verfügbaren Umwelterklärungen (ca. 1100) und -berichte (ca. 250) wurden, bezogen auf das Jahr 1995 bzw. 1995/96, die 498 Umwelterklärungen und 55 Umweltberichte als auswertbare Datenbasis genommen, die auf ein Anschreiben mit der Rücksendung einer Umwelterklärung oder eines -berichtes geantwortet haben. Vgl. für eine ausführliche Darstellung der Ergebnisse Hroch, N./ Schaltegger, S.: Wie aktuell sind Umwelterklärungen und -berichte bezüglich der umweltpolitischen Diskussion? Untersucht am Beispiel von Angaben über C02-Emissionen und Energieverbrauch, INFU-Diskussionsbeiträge 9/1999, Institut für Umweltkommunikation der Universitüt Lüneburg. (2) Der Wert ergibt sich als 370/3318 $=11,15$ Prozent. Die Zahl von 3318 maximalen Nennungen ergibt sich aus der Multiplikation der Anzahl untersuchter Kriterien (6) mit der Anzahl von Veröffentlichungen (553).

(3) Vgl. Europäische Gemeinschaften: Verordnung (EWG) Nr. 1836/93 des Rates vom 29. Juni 1993 über die freiwillige Beteiligung gewerblicher Unternehmen an einem Gemeinschaftssystem für das Umweltmanagement und die Umweltbetriebsprüfung.

(4) Eine entsprechende Forfführung des Projekts mit der Untersuchung von Umweltberichten und -erklärungen, bezogen auf das Jahr 1998/99, läuft derzeit. Mit dieser Panelanalyse soll sowohl ein Quer- als auch ein Zeitreihenvergleich ermöglicht werden.

\section{Die Autorlnnen}

Nicole Hroch ist wissenschaftliche Mitarbeiterin, Dr. Stefan Schaltegger ist Professor am Lehrstuhl für Betriebswirtschaftslehre (Umweltmanagement), an der Universität Lüneburg sowie Mitglied des VÖW-Vorstands. Kontakt: Universität Lüneburg, Scharnhorststr. I, D-21335 Lüneburg. E-mail: hroch@uni-lueneburg.de 
(c) 20I0 Authors; licensee IÖW and oekom verlag. This is an article distributed under the terms of the Creative Commons Attribution Non-Commercial No Derivates License (http://creativecommons.org/licenses/by-nc-nd/3.o/), which permits unrestricted use, distribution, and reproduction in any medium, provided the original work is properly cited. 